\title{
Performance and Molière radius measurements using a compact prototype of LumiCal in an electron test beam
}

\author{
H. Abramowicz ${ }^{1}$, A. Abusleme ${ }^{2}$, K. Afanaciev ${ }^{3}$, Y. Benhammou ${ }^{1}$, O. Borysov ${ }^{1}$, M. Borysova ${ }^{1,13}$, \\ I. Bozovic-Jelisavcic ${ }^{4}$, W. Daniluk ${ }^{5}$, D. Dannheim ${ }^{6}$, M. Demichev ${ }^{7}$, K. Elsener ${ }^{6}$, M. Firlej ${ }^{8}$, E. Firu ${ }^{9}$, T. Fiutowski ${ }^{8}$, \\ V. Ghenescu ${ }^{9}$, M. Gostkin ${ }^{7}$, M. Hempel ${ }^{10,14}$, H. Henschel ${ }^{10}$, M. Idzik ${ }^{8}$, A. Ignatenko ${ }^{3,15}$, A. Ishikawa ${ }^{11}$, A. Joffe ${ }^{1}$, \\ G. Kacarevic ${ }^{4}$, S. Kananov ${ }^{1}$, O. Karacheban ${ }^{10,14}$, W. Klempt ${ }^{6}$, S. Kotov ${ }^{7}$, J. Kotula ${ }^{5}$, U. Kruchonak ${ }^{7}$, Sz. Kulis ${ }^{6}$, \\ W. Lange ${ }^{10}$, J. Leonard ${ }^{10}$, T. Lesiak ${ }^{5}$, A. Levy ${ }^{1, a}$, I. Levy ${ }^{1}$, L. Linssen ${ }^{6}$, W. Lohmann ${ }^{10,14}$, J. Moron ${ }^{8}$, \\ A. Moszczynski ${ }^{5}$, A. T. Neagu ${ }^{9}$, B. Pawlik ${ }^{5}$, T. Preda ${ }^{9}$, A. Sailer ${ }^{6}$, B. Schumm ${ }^{12}$, S. Schuwalow ${ }^{10,16}$, E. Sicking ${ }^{6}$, \\ K. Swientek ${ }^{8}$, B. Turbiarz ${ }^{5}$, N. Vukasinovic $^{4}$, T. Wojton $^{5}$, H. Yamamoto ${ }^{11}$, L. Zawiejski ${ }^{5}$, I. S. Zgura ${ }^{9}$, \\ A. Zhemchugov ${ }^{7}$
}

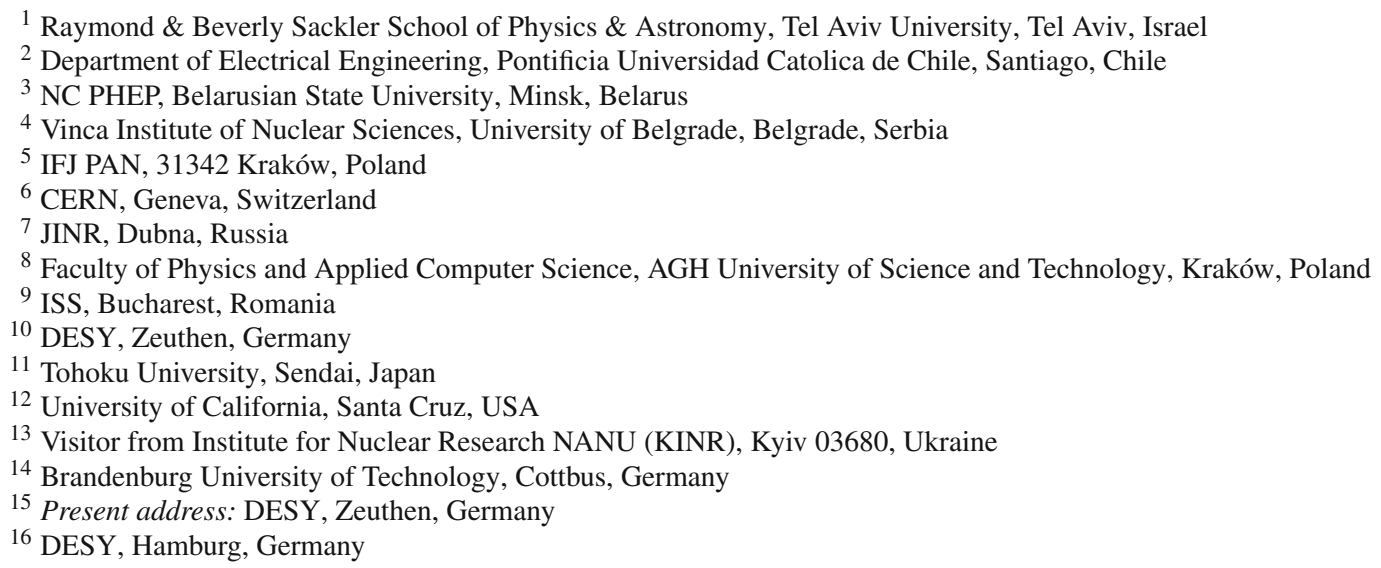

\begin{abstract}
A new design of a detector plane of submillimetre thickness for an electromagnetic sampling calorimeter is presented. It is intended to be used in the luminometers LumiCal and BeamCal in future linear $\mathrm{e}^{+} \mathrm{e}^{-}$collider experiments. The detector planes were produced utilising novel connectivity scheme technologies. They were installed in a compact prototype of the calorimeter and tested at DESY with an electron beam of energy $1-5 \mathrm{GeV}$. The performance of a prototype of a compact LumiCal comprising eight detector planes was studied. The effective Molière radius at $5 \mathrm{GeV}$ was determined to be $(8.1 \pm 0.1$ (stat) \pm 0.3 (syst)) mm, a value well reproduced by the Monte Carlo (MC) simulation $(8.4 \pm 0.1) \mathrm{mm}$. The dependence of the effective Molière radius on the electron energy in the range 1-5 GeV was also studied. Good agreement was obtained between data and MC simulation.
\end{abstract}

\section{Introduction}

Forward calorimeters for future electron positron linear collider experiments have challenging requirements on a fast and high precision measurement of the luminosity [1], resulting in a stringent set of specifications for highly compact calorimeters. Two such calorimeters, LumiCal and Beam$\mathrm{Cal}$, are being considered for installation in the forward region of both International Linear Collider (ILC) [2,3] detectors, ILD and $\mathrm{SiD}$, and also in the Compact Linear Collider (CLIC) detector [4]. The precise measurement of the integrated luminosity is provided by the LumiCal detector. BeamCal is designed for instant luminosity measurement and beam-tuning when included in a fast feedback system as well as for tagging beam particles scattered through low angles. Both detectors extend the capabilities of the experiments for physics studies in the high rapidity region.

a e-mail: levyaron@tauex.tau.ac.il 


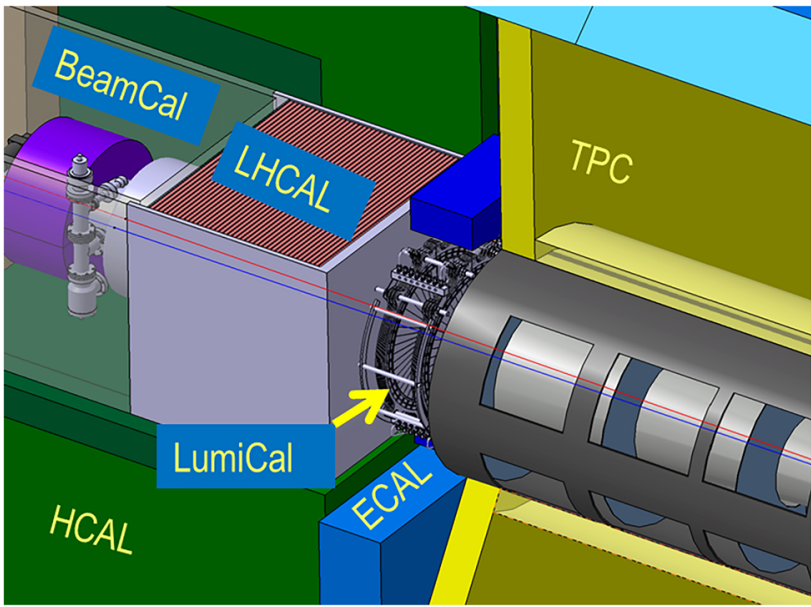

Fig. 1 The very forward region of the ILD detector. LumiCal, Beam$\mathrm{Cal}$ and LHCAL are carried by the support tube for the final focusing quadrupole and the beam-pipe. TPC, ECAL and HCAL are the Time Projection Chamber and the Electromagnetic and Hadron Calorimeter

The layout of one arm of the forward region of the ILD detector is presented in Fig. 1. LumiCal is positioned in a circular hole of the end-cap electromagnetic calorimeter ECAL. BeamCal is placed just in front of the final focus quadrupole. LumiCal is designed as a sampling calorimeter composed of 30 layers of $3.5 \mathrm{~mm}\left(1 \mathrm{X}_{0}\right)$ thick tungsten absorbers and silicon sensors placed in a one-millimeter gap between absorber plates. BeamCal has a similar design as LumiCal. For the current BeamCal baseline design, GaAs sensors are considered which can withstand higher radiation doses at room temperature. The similarity between LumiCal and BeamCal designs implies that the technology developed for one can be used also for the other.

Luminosity in LumiCal is measured using Bhabha scattering, $\mathrm{e}^{+} \mathrm{e}^{-} \rightarrow \mathrm{e}^{+} \mathrm{e}^{-}(\gamma)$, as a gauge process. The Bhabha scattering cross section can be precisely calculated in QED [5] and the luminosity, L, is obtained as

$\mathrm{L}=\frac{N_{\mathrm{B}}}{\sigma_{\mathrm{B}}}$,

where $N_{\mathrm{B}}$ is the number of Bhabha events registered by LumiCal in a given range of polar angles $\left(\theta_{\min }, \theta_{\max }\right)$ and $\sigma_{\mathrm{B}}$ is the integral of the differential cross section over the same range. This range defines the fiducial volume of the calorimeter. The fiducial volume for the LumiCal baseline design was studied in simulations [1] and found to be in the range from 41 to $67 \mathrm{mrad}$ while the geometrical coverage of the LumiCal ranges from 31 to $77 \mathrm{mrad}$. The fiducial volume is reduced due to the lateral energy leakage which depends on the electromagnetic shower development in the transverse plane. The compact design of the LumiCal with small gaps between absorber plates allows the transverse size of the shower to be kept small and to achieve in a relatively small $\theta$ angle range a sufficiently large fiducial volume for a precise luminosity measurement. It also improves the efficiency to detect electrons on top of a widely-spread background originating from beamstrahlung and two-photon processes.

In addition, the compact construction of LumiCal and BeamCal are essential to match the strict geometrical constraints imposed by the design of the detectors and accelerator needs near the interaction point.

In an earlier test beam of a four-layer silicon-tungsten prototype of the LumiCal, an effective Molière radius ${ }^{1}$ of $24.0 \pm 0.6 \mathrm{~mm}$ was measured [6]. The reason for this large value was a large air gap between the silicon sensor plane and the absorber plates because space was needed for a $3.5 \mathrm{~mm}$ thick readout board.

In order to get a smaller Molière radius, it was essential to design, build and use planes of sub-millimetre thickness to be inserted in a mechanical frame [7] in one millimetre gaps between the tungsten absorber plates.

This paper describes the design and construction of a compact LumiCal prototype calorimeter, hereafter referred to as calorimeter, and the results from test-beam measurements carried out at DESY, using an electron beam between 1 and $5 \mathrm{GeV}$ energy. For the readout electronics, APV25 front-end boards [8-10] were used. The effective Molière radius of this compact configuration was calculated in a similar way to that in Ref. [6]. The energy dependence of the effective Molière radius in the energy range of $1-5 \mathrm{GeV}$ is also measured. In addition, two sensor planes were put in front of the calorimeter to serve as tracker planes to distinguish between electrons and photons. The results of this latter study will be presented elsewhere.

\section{Thin detector plane construction}

The design of a LumiCal sensor was optimised in simulations to provide the required resolution of the polar angle reconstruction. A picture of a sensor is shown in Fig. 2. The sensor is made of a $320 \mu \mathrm{m}$ thick high resistivity n-type silicon wafer. It has the shape of a sector of a $30^{\circ}$ angle, with inner and outer radii of the sensitive area of $80 \mathrm{~mm}$ and $195.2 \mathrm{~mm}$, respectively. It comprises four sectors with 64 p-type pads of $1.8 \mathrm{~mm}$ pitch.

The properties of the sensor were studied in the lab and beam tests. Results of beam tests and more details about the sensor can be found in Refs. [6,11]. The first prototype of a LumiCal detector plane, which has been successfully used in a multi-layer configuration [6], had a thickness of about $4 \mathrm{~mm}$ and only 32 pads were connected to the readout electronics.

\footnotetext{
1 As we do not have a fully contained shower in the prototype of LumiCal used in the earlier and also this test beam, we measure an effective Molière radius.
} 


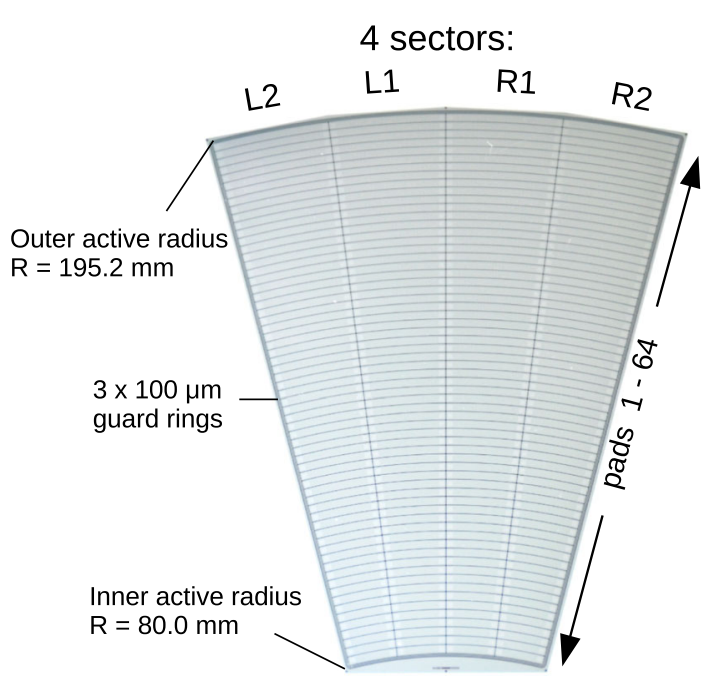

Fig. 2 A LumiCal silicon sensor

For the construction of a sub-millimetre detector plane we used the same silicon sensor. The bias voltage is supplied to the $\mathrm{n}$-side of the sensor by a $70 \mu \mathrm{m}$ flexible Kapton-copper foil, glued to the sensor with a conductive glue. The 256 pads of the sensor are connected to the front-end electronics using a fan-out made of $120 \mu \mathrm{m}$ thick flexible Kapton foil with copper traces. The inner guard ring is grounded. Ultrasonic wire bonding was used to connect conductive traces on the fanout to the sensor pads. A support structure, made of carbon fibre composite with a thickness of $100 \mu \mathrm{m}$ in the sensorgluing area, provides mechanical stability for the detector plane. Special fixtures were designed and produced to ensure the necessary thickness and uniformity of three glue layers between different components of the detector plane all over the area of the sensor. A sketch of the structure of the detector plane is shown in Fig. 3 and a photo of a completed plane in Fig. 4. Since the multi-channel version of the dedicated front-end electronics is still under development, the APV25 front-end board [8,9], used by the silicon strip detector of the CMS experiment, was chosen as a temporary solution. It has 128 channels, hence two boards read the whole sensor.

The ultrasonic wire bonding proved to provide good electrical performance, but for a detector plane thinner than $1 \mathrm{~mm}$, the wire loops, which are typically 100-200 $\mu \mathrm{m}$ high, cause a serious problem when the plane needs to be installed in a $1 \mathrm{~mm}$ gap between absorber plates. The parameters of the bonding machine were studied and tuned to make the loop as low as possible and technically acceptable. The sampling based measurements, which were done using a con-focal laser scanning microscope, show that the loop height is in the range from 50 to $100 \mu \mathrm{m}$.

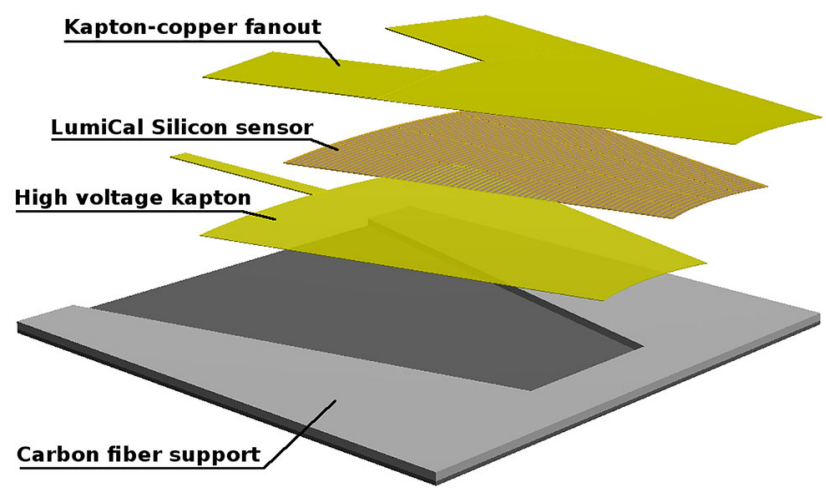

Fig. 3 Detector plane assembly. The thickness of adhesive layers (not shown) between components is within $10-15 \mu \mathrm{m}$. The total thickness is $650 \mu \mathrm{m}$

\section{Beam test setup}

The detector planes were installed in the 1-mm gap between the tungsten absorber layers. Each tungsten absorber layer is on average $3.5 \mathrm{~mm}$ thick and roughly one radiation length $\left(1 X_{0}\right)$. As described in Fig. 5, the first calorimeter sensor layer was placed after 3 absorber layers, and the rest followed after each additional absorber layer. The last sensor layer was placed after 8 absorber layers with a total thickness of $7.7 X_{0}$, since, as noted in [6], the absorber layers are not pure tungsten. The detector planes were tested in two beam test campaigns in 2015 and 2016 at the DESY-II Synchrotron using electrons with energies between 1 and $5 \mathrm{GeV}$.

The beam test aimed to study the performance of the compact calorimeter and to test the concept of tracking detectors in front of the calorimeter as a tool for electron and photon identification. The geometry of the setup is shown in Fig. 5.

The electron beam passed through a $5 \times 5 \mathrm{~mm}^{2}$ square collimator that limits the beam spread along the test setup. The AIDA/EUDET beam telescope was placed upstream of the calorimeter. The telescope was split into two parts T1 and T2, each containing an arm with 3 layers of MIMOSA26 pixel silicon detectors and 2 thin scintillator counters Sc1 and Sc2, for the trigger system. The telescope front arm was placed before the dipole magnet to record the incoming electrons. The rear arm was placed after the dipole magnet to record the electrons in the direction of the calorimeter, and to separate them from the photons generated in the copper target that was mounted just in front of the magnet.

The calorimeter and tracker were assembled in a mechanical frame [7] specially designed to provide high precision positioning of the sensor planes and absorber plates. The sensor planes are attached to the tungsten absorber plates 


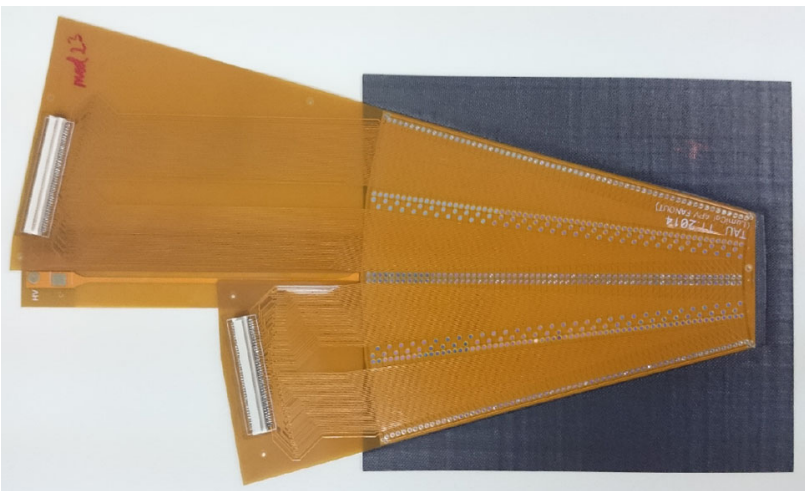

Fig. 4 A thin detector plane. The black part is the carbon fibre support. The silicon sensor is covered by the Kapton fan-out which has two connectors for front-end boards

by adhesive tape. The tungsten plates are glued to permaglass inserted into the comb slots of the mechanical structure. The assembly of the calorimeter is illustrated in Fig. 6. Two sub-millimetre planes, viewed separately in the upper part of Fig. 6, are the tracker planes denoted as "Tracker" in Fig. 5. They are installed in front of the calorimeter.

The last module in the LumiCal stack shown in grey is assembled using the tape automatic bonding (TAB) technology [12]. This sensor plane was not used in the present analysis. All detector planes, for both the calorimeter and the tracker, were powered with a reverse bias voltage of $120 \mathrm{~V}$. This bias voltage is about 2 to 3 times the depletion voltage [11], but well below the breaking voltage of these silicon sensors.

\section{Data acquisition}

A sketch of the data acquisition system (DAQ) is shown in Fig. 7. It comprises two interdependent systems. The first one is the EUDAQ which controls the beam Telescope and

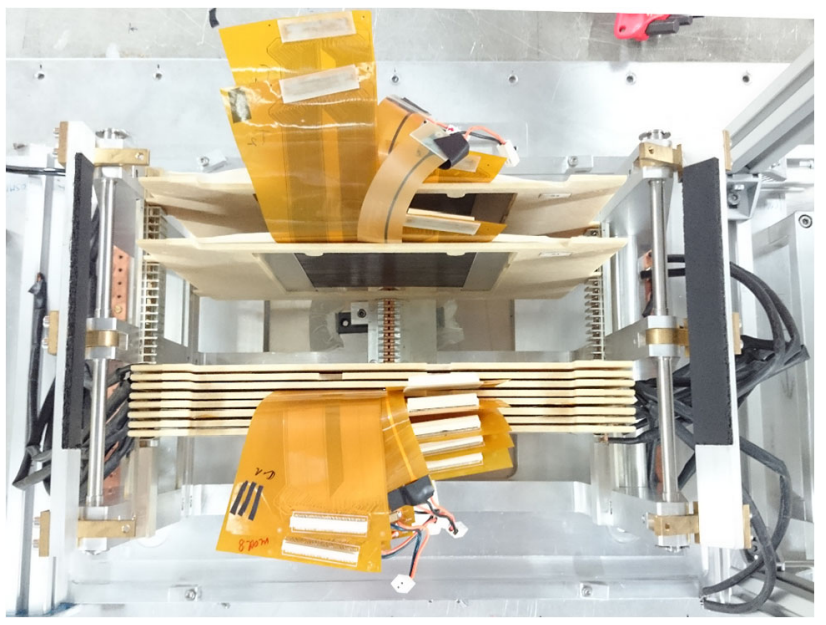

Fig. 6 Top view of the assembled calorimeter

the Trigger Logic Unit, TLU. The second, the calorimeter DAQ, is based on the Scalable Readout System (SRS) [13], developed by the RD51 collaboration, and described below. A trigger signal is generated in the TLU, as a coincidence of signals from the scintillator counters $\mathrm{Sc} 1$ and $\mathrm{Sc} 2$, both consisting of two thin scintillators with attached photomultipliers. The TLU then sends the trigger signal to both the Telescope acquisition and to the SRS. In addition, a BUSY signal is provided by a NIM logic to prevent the TLU from sending more signals before the event acquisition ended. The SRS, with a front-end hybrid board [10] based on the APV25 front-end chip, is used for the readout. The APV25 front-end board has 128 readout channels, each consisting of a charge sensitive preamplifier and a shaper with a CR-RC filter producing a $50 \mathrm{~ns}$ shaped voltage pulse $[8,9]$. The output of the shaper is sampled at $40 \mathrm{MHz}$ and stored in an analog pipeline. During the beam test, the APV25 front-end boards are configured to operate in multi-mode, transmitting, upon receipt of a trigger from the TLU, 21 consecutive pipeline samples of each channel to the adapter board of the SRS through 3-

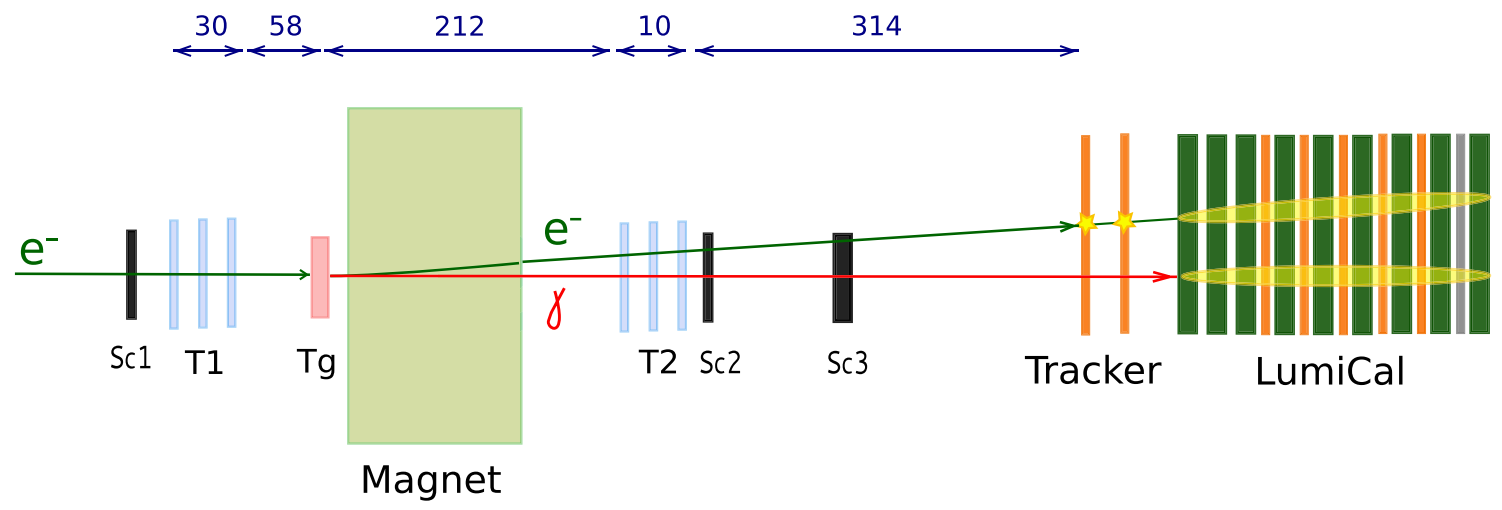

Fig. 5 Geometry of the beam test setup (not to scale). Sc1, Sc2 and $\mathrm{Sc} 3$ are scintillator counters; $\mathrm{T} 1$ and $\mathrm{T} 2$ the arms of three-pixel detector planes, $\mathrm{Tg}$ the copper target for bremsstrahlung photon production and
LumiCal, the calorimeter prototype under test. Distances, rounded to integer numbers in centimetres, are shown in the upper part of the figure 


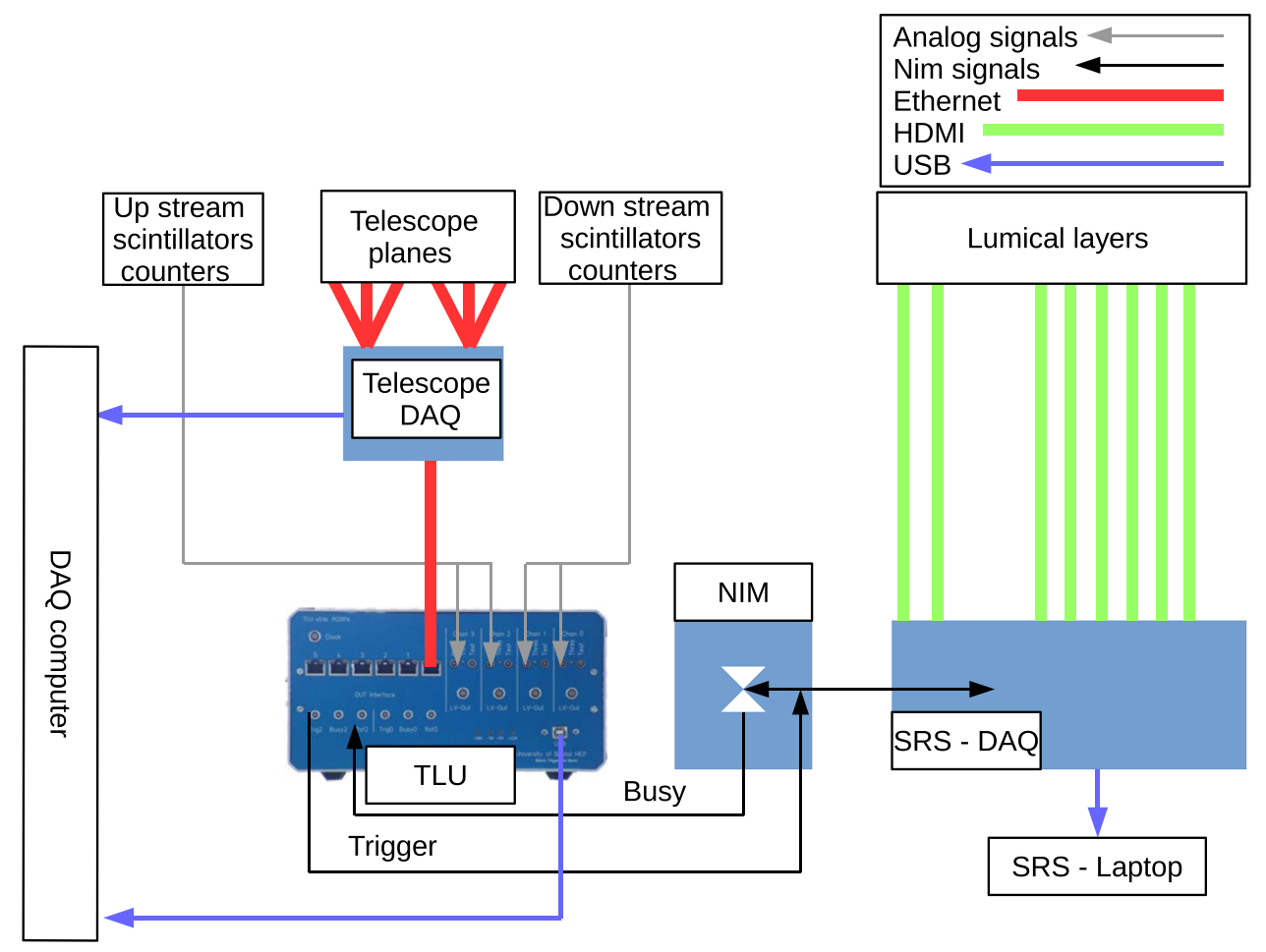

Fig. 7 The data acquisition system

m long HDMI cables. These samples are converted to 12-bit numbers in the SRS adapter board and transmitted to the data acquisition PC.

Simulation results for the present configuration show that a single pad in a shower can be hit by 80 relativistic particles, hereafter referred to as MIPs 2 (see Fig. 13 in Sect. 6). The usage of the APV25 front-end board, which has a dynamic range for energy depositions originating from up to 8 MIPs, is hence not appropriate to read out sensor pads inside an electromagnetic shower. In order to enable measurements of a wider range of deposited energies, a capacitive charge divider is connected to the input of the APV25 front-end board. The attenuation factor of the charge divider is optimised by using the results from MC simulation. However, small signals from pads with low energy depositions in the tails of the shower are then below the detection threshold. The simulation of the observed noise level and the geometry of the present calorimeter shows that an attenuation of the signal with a factor of 3.5-4.5, results in a 5-7\% loss of the deposited energy, which can be corrected for as described in Sect. 6 .

\section{Signal processing}

The APV25 front-end chip operating in the multi-mode provides readout of 21 consecutive pipeline samples. The baseline of the output for each channel is calculated as the average of these samples in a dedicated pedestal run with a random trigger without beam. The noise is estimated as the standard deviation of the samples in the pedestal run and is used for setting the threshold in data during the run with a beam. An example of the signal for a single channel, after baseline and common-mode noise subtraction, is shown in Fig. 8. During data taking, the average of 21 samples of each channel is calculated and compared to the zero suppression (ZS) threshold. If it is below the ZS threshold, the data for the channel is not recorded. The threshold is set to 0.4 times the channel noise which results in a low enough threshold not to reject the signal from particles. Data is collected asynchronously, i.e. the readout electronics is not synchronised with the accelerator clock. As a consequence, most of the time the signal is not sampled exactly at its maximum. To determine the signal maximum, the samples are fitted with a CR-RC filter response function, as shown in Fig. 8,

$S(t)=A \frac{t-t_{0}}{\tau} e^{-\frac{t-t_{0}}{\tau}} \Theta\left(t-t_{0}\right)$,

where $t_{0}$ is the arrival time of the signal, $\tau=50 \mathrm{~ns}$ is the peaking time of the APV25 front-end board and $A$ is the relative signal amplitude. The function $\Theta\left(t-t_{0}\right)$ is the Heaviside step function.

The relatively low ZS threshold allows a significant amount of noise pulses to pass through and further signal selection criteria are applied in the analysis. First, an artificial neural network (ANN) is used to analyse the signal and classify the data based on its shape. The ANN is represented by multilayer perceptron model with 21 inputs fed 


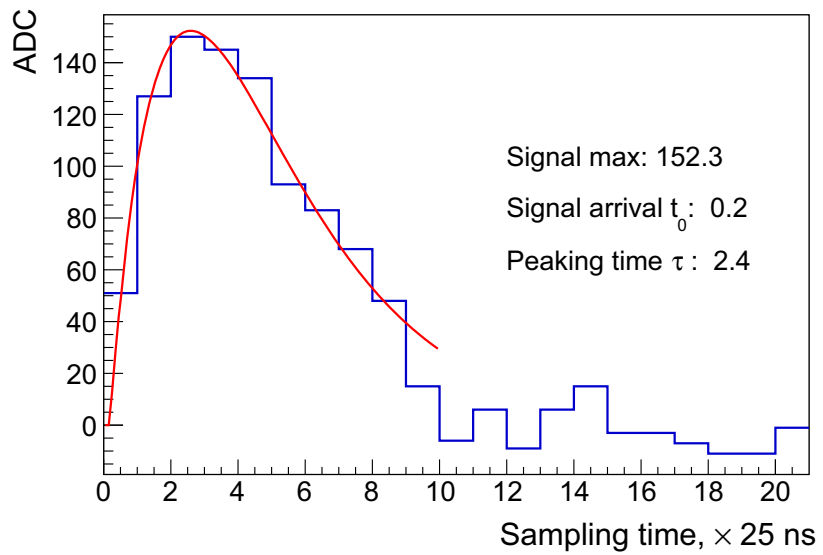

Fig. 8 Signal waveform sampled in $25 \mathrm{~ns}$ time intervals. The red line is a fit with CR-RC-response function of Eq. (2)

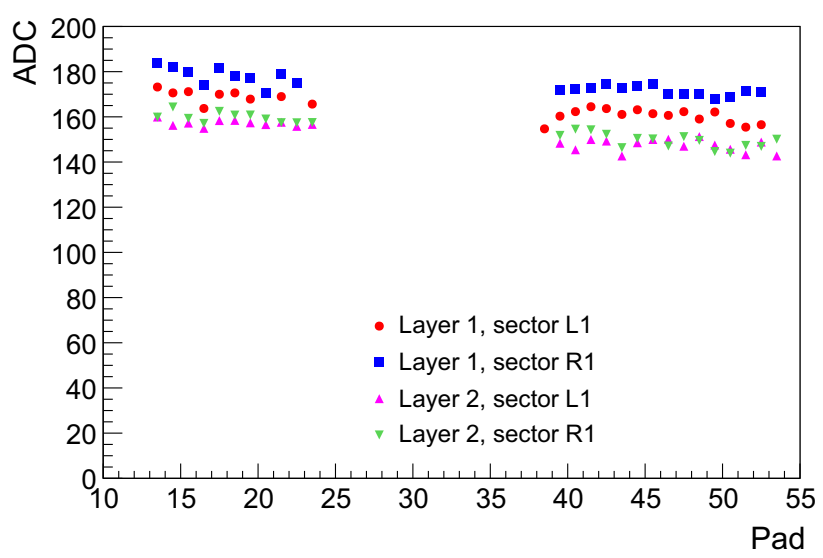

Fig. 10 Most probable value of the signal in the pads of the tracking layers covered by the electron beam of $5 \mathrm{GeV}$

from the APV25 samples and one hidden layer with 10 nodes. The training set for different signal amplitudes is generated using the function in Eq. (2) with a Gaussian noise added to each sample. After signal preselection based on the ANN, the signal is fitted with Eq. (2), where the amplitude, arrival time $t_{0}$ and peaking time $\tau$ are used as parameters. To further improve the purity of the signal, selection criteria are applied to the parameters $t_{0}$ and $\tau$. The efficiency of the selection is studied using external pulses, as described in Sect. 6.

Figure 9 shows the distribution of the signal amplitudes produced by a $5 \mathrm{GeV}$ electron beam and measured in a single channel of the tracking plane. The green line corresponds to the data which pass the ZS threshold. The blue line, which corresponds to the data after applying additional signal selection criteria, illustrates the effective noise suppression in the analysis. The most probable value (MPV) of the peak is estimated using a fit with a convolution of Landau and Gaussian

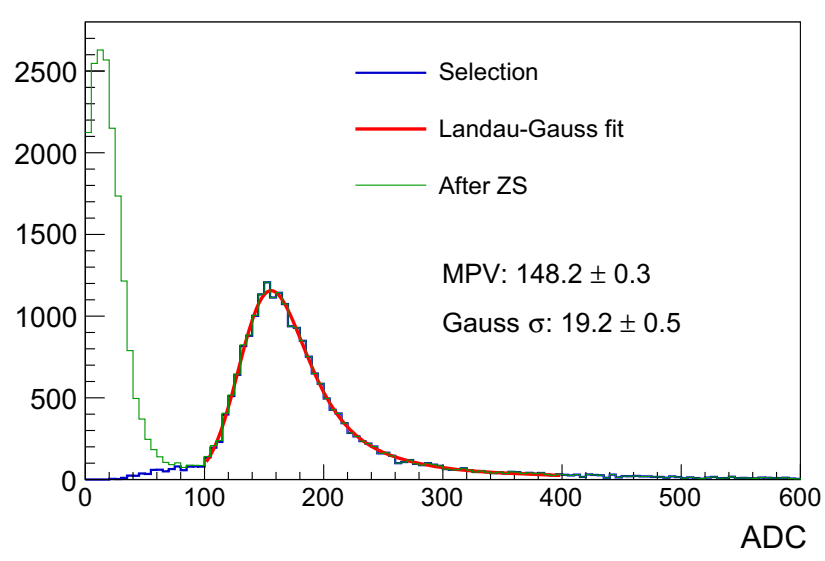

Fig. 9 Signal distribution in a single pad of the tracking layer. Green line - after zero suppression, blue line - after additional selection criteria, and red line - fit with a convolution of Landau and Gaussian distribution functions

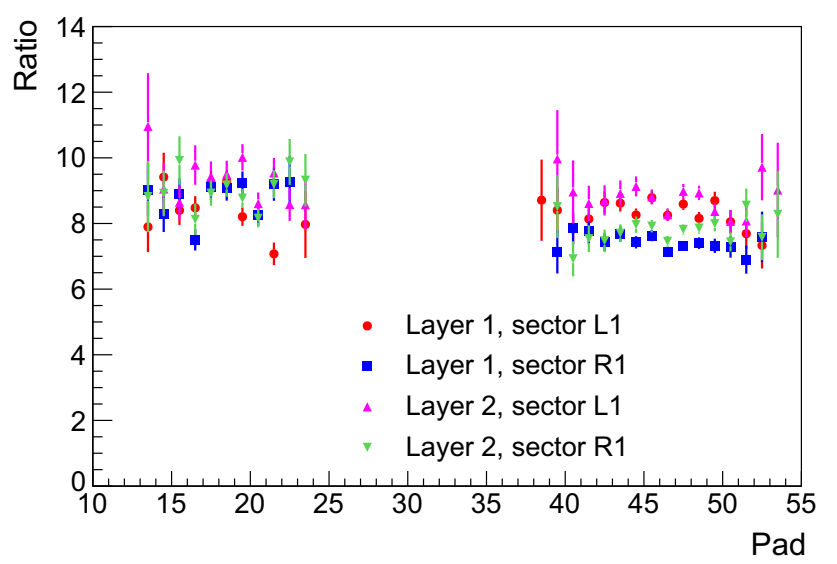

Fig. 11 Signal to noise ratio for the pads of the tracking layers covered by the electron beam of $5 \mathrm{GeV}$

distribution functions. The width $\sigma$ of the Gaussian distribution is considered as noise measurement. The MPV values of the amplitude distribution corresponding to $5 \mathrm{GeV}$ electrons are shown in Fig. 10. The higher values for small pad numbers reflect the geometry of the sensor where these pads have smaller area and smaller capacitance. The same effect is observed for the signal-to-noise ratio shown in Fig. 11. Since the beam profile has blurry edges, the statistical uncertainties increase for pads that correspond to the periphery of the beam. For most of the channels the signal-to-noise ratio is within a range from 7 to 10 . The most probable value of the energy deposited by $5 \mathrm{GeV}$ electrons is used to define the unit MIP for the energy deposition in the sensors. Based on MC simulations, a MIP corresponds to $88.5 \mathrm{keV}$.

For the detector planes that are installed in the calorimeter, the capacitive charge divider is used. The signals from single particles are too small to be registered, and hence the signal- 


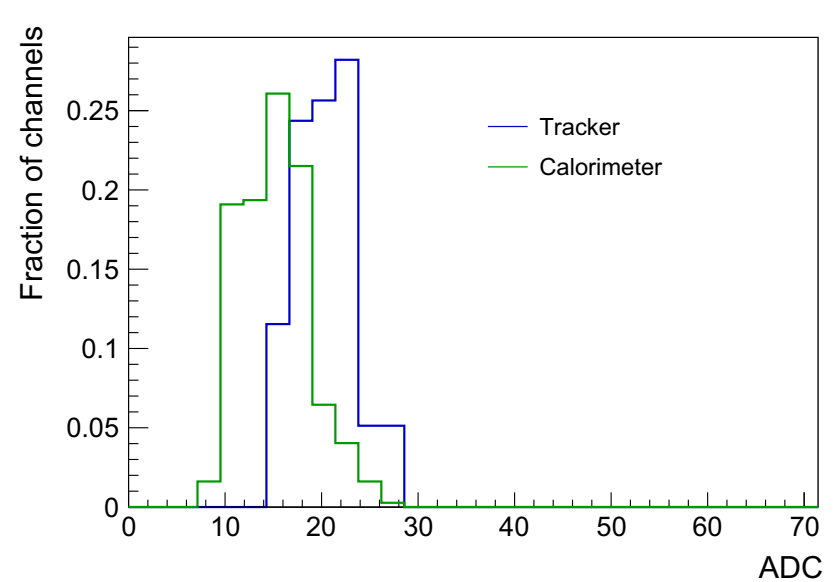

Fig. 12 Noise distribution in the channels of the tracking layers (blue) and the calorimeter layers (green)

to-noise ratio cannot be measured. Taking into account the design of the charge divider and the noise measured in the pedestal run, as shown in Fig. 12, the estimated value of signal-to-noise ratio is in the range of 2-3. For such a low ratio, the signal-shape analysis, using ANN and selectioncriteria for the parameters retrieved from the fit, allows for the efficient identification of the signal with little contamination from noise.

\section{Calibration of the APV25 front-end board}

The linearity of the APV25 front-end boards was studied with the bare chip $[8,9]$ and it was found to be very good for signals of up to 3 MIPs and remains better than $5 \%$ up to 5 MIPs.

The relative response of the APV25 channels, equipped with a capacitive charge divider, is measured using a voltage pulse supplied to the channel input through a capacitor of $2 \mathrm{pF}$. The detector capacitance is simulated by a $7 \mathrm{pF}$ capacitor connected in parallel to the channel input. About 10 randomly chosen channels for each APV25 front-end chip were measured and the average response curve was calculated for each APV25.

The APV25 front-end board with charge divider approaches saturation at about 1600 ADC counts. In this analysis, the maximum signal size is 1450 ADC counts, reasonably below the saturation.

Figure 13 shows the distribution of the deposited energy in a pad in the detector layer after 5 tungsten plates. The data were processed with the calibration obtained by interpolation between measured values. The sharp spikes are due to saturation which, after calibration, has slightly different thresholds for each APV25. The measured distribution of the deposited energy in a single pad is well reproduced by the simulations for signal amplitudes larger than 5 MIPs. However, smaller signals become masked by the noise. This loss of signals can also be seen in Fig. 13, where for small amplitudes the experimental distribution is below the MC expectation.

In order to correct for this loss of signals, the efficiency $\epsilon$ of identifying the signal of a small amplitude is studied with the same setup using an external voltage pulse. We define the efficiency of signal identification as the ratio of the number of identified signals to the number of generated ones. This ratio depends on the signal-to-noise ratio and therefore is slightly different for different APV25 front-end chips, as shown in Fig. 14 where the results for channels of different APV25 front-end chips are presented. For each APV25 front-end chip, about 10 channels are measured. For signals larger than 10 MIPs, the efficiency is $100 \%$ in all channels. For a smaller number of MIPs, some channels give lower efficiencies. The measurements of the efficiency $\epsilon$ are fit by the following expression:

$\epsilon=p_{0}\left(1+\operatorname{erf}\left(\frac{S-S_{0}}{p_{1}}\right)\right)$

where $\operatorname{erf}$ is the error function, $S$ the signal amplitude and $p_{0}, p_{1}$ and $S_{0}$ are fit parameters. The red curve in Fig. 14 represents the average of the fit of a large number of channels and the shaded area the spread of the fit in these channels at low signal amplitudes. Since the noise level observed during lab calibration measurements and beam test are similar, the efficiency correction for small signal sizes is applied to the test-beam simulations using the results of the fit to Eqn. (3).

\section{Results}

More than seven million events were collected in an electron beam from 1 to $5 \mathrm{GeV}$ energy, with $1 \mathrm{GeV}$ steps, for different setup configurations to measure the precision of the shower position determination, the electromagnetic shower development in longitudinal and transverse directions and the effective Molière radius.

Figure 15 shows the distributions of the energy deposited in the sensors of the calorimeter for beam electrons of different energy. The average deposited energy as a function of the electron beam energy is presented in Fig. 16. The measured raw values increase with increasing beam energy, with a tendency of a reduced slope at larger beam energies. After applying the APV25 calibration, as described in Sect. 6, and correcting for the energy leakage fraction, estimated from the simulation, the response becomes nearly linear.

\subsection{Reconstruction of the shower position}

For the reconstruction of the shower position, pads with deposited energy were combined into clusters. In the first 


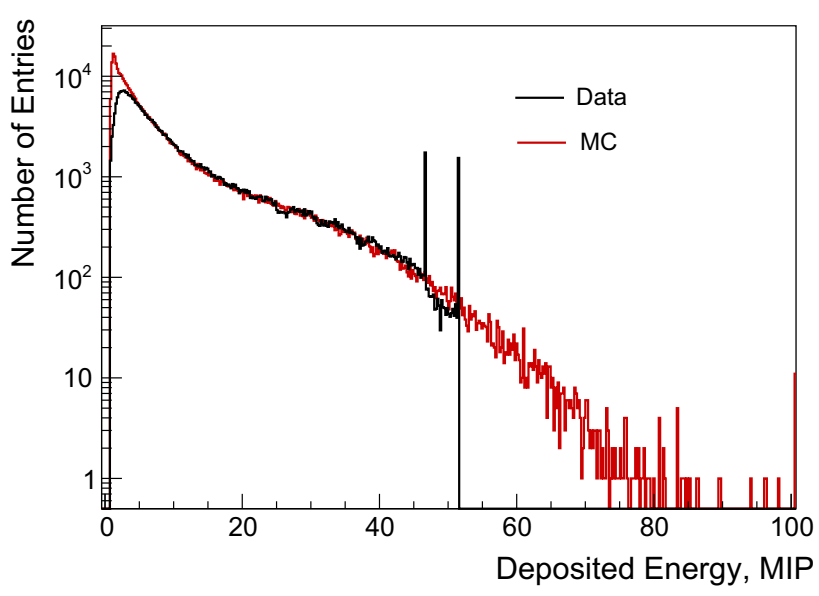

Fig. 13 Distribution of the deposited energy in a sensor pad in the detector layer after 5 tungsten plates. The red line is a MC simulation, and the black line is data using as calibration an interpolation between measured calibration values of the APV25 front-end chip. The sharp spikes are due to saturation in two APV25 front-end boards

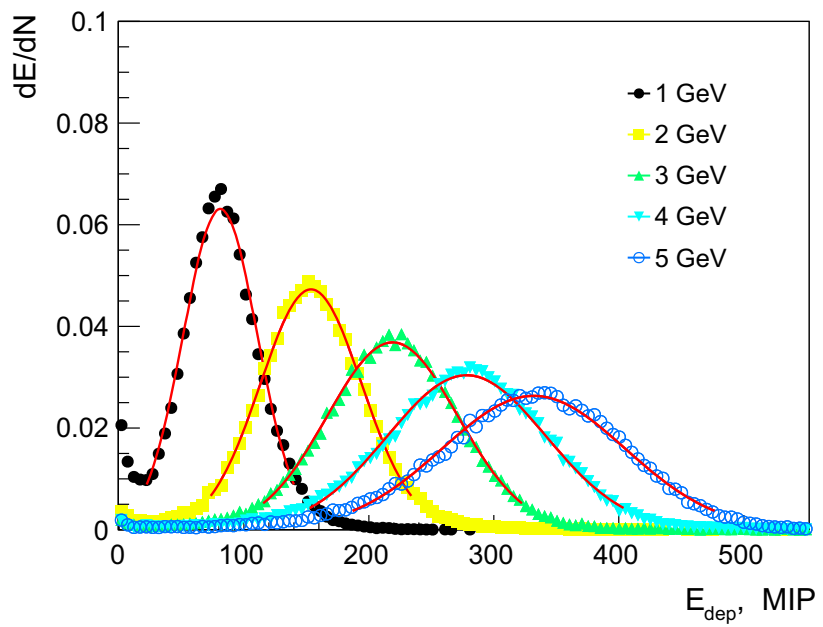

Fig. 15 Distribution of the energy deposited in the calorimeter, $d E / d N$, for different electron energies. The lines illustrate fits with a Gaussian

step, the depositions in all pads at a given radial and azimuthal position are summed over all detector layers. The clustering algorithm used in this study builds a cluster including all nearest neighbour pads. The pad with radial number $n$ and sector number $k$ is assigned to a cluster if the cluster contains a pad with radial number $n^{\prime}$ and sector numbers $k^{\prime}$ such that both $\left|n-n^{\prime}\right| \leq 1$ and $\left|k-k^{\prime}\right| \leq 1$. If this holds, the cluster is considered as an electromagnetic shower. The shower position is determined using a weighted sum:

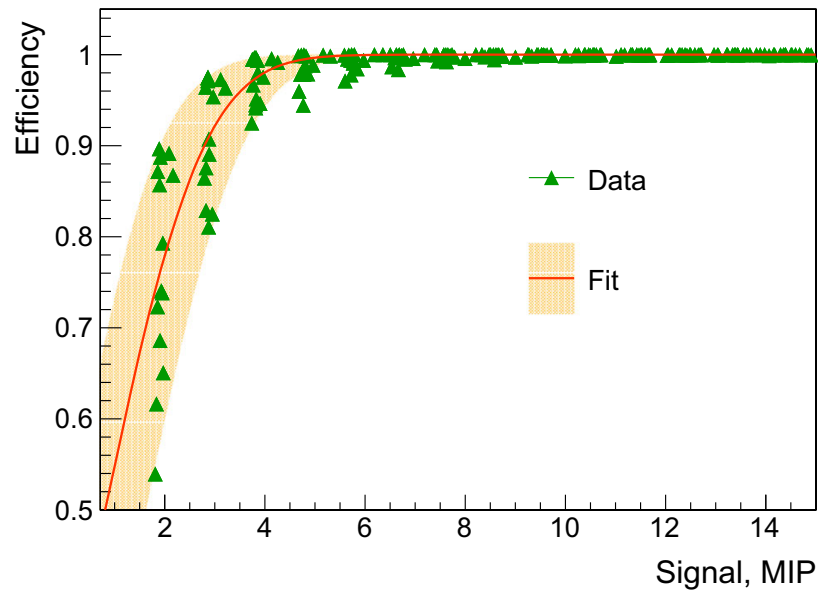

Fig. 14 Efficiency of signal identification as a function of the signal amplitude. Green triangles are measured for different channels, the red line is an average of the fit using Eq. (3) to a large number of channels, and the shaded area corresponds to the spread of fits at small amplitudes

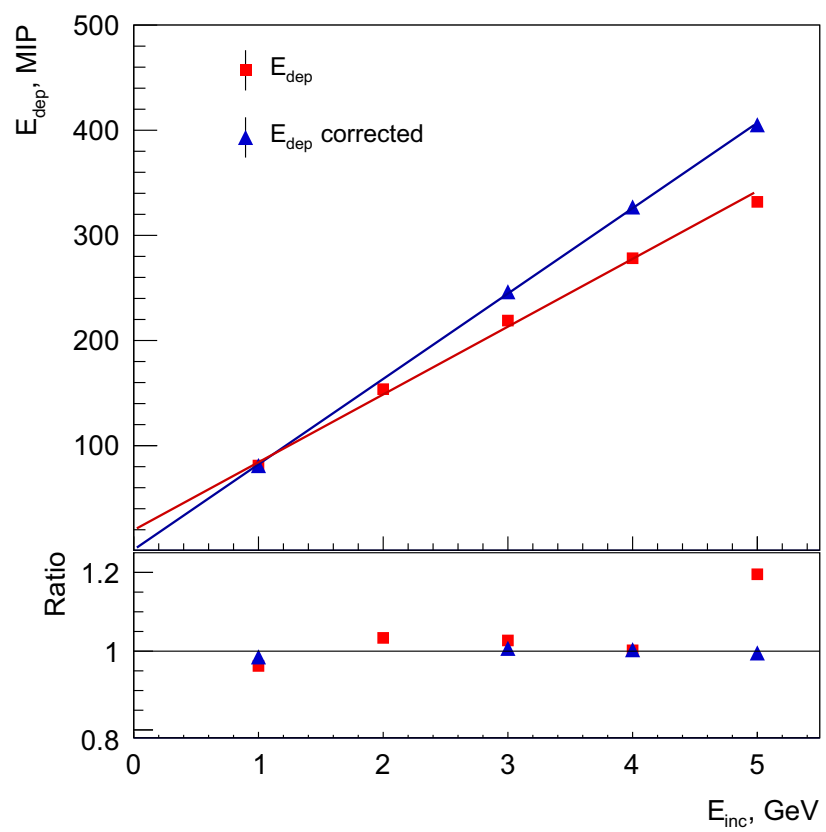

Fig. 16 Average deposited energy in the calorimeter, $E_{d e p}$, as a function of beam energy before (red) and after applying the APV25 calibration and corrections to the leakage fraction estimated from the simulation (blue). The lines are straight line fits to the data. The lower part of the figure shows the ratio of the deposited energy to the straight line fit

$Y_{c}=\frac{\sum_{m} Y_{m} w_{m}}{\sum_{m} w_{m}}$

where the index $m$ runs over all pads included in the shower. $Y_{m}$ is the position of the pad and $w_{m}$ is a weight, which in the simplest approach could be taken as the energy $E_{m}$ deposited in the pad. It has however been shown [14-16] that this approach gives a biased estimate when the shower 


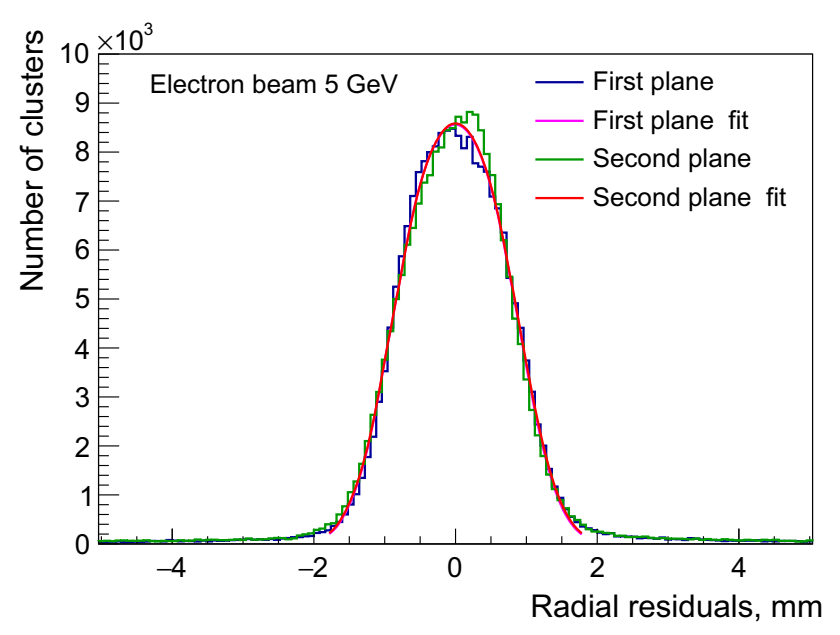

Fig. 17 Distribution of residuals of the radial position measurements in the tracking planes and the calorimeter, obtained with a $5 \mathrm{GeV}$ electron beam

position is not in the centre of a pad. Several methods were developed to achieve more accurate position reconstruction, and the following choice of weights is found to be the most appropriate:

$w_{m}=\max \left\{0 ; W_{0}+\ln \frac{E_{m}}{\sum_{j} E_{j}}\right\}$,

where $W_{0}$ is a free dimensionless parameter. The performance of the clustering algorithm is studied in a simulation and for the present configuration the best resolution for the radial coordinate of the shower is achieved with $W_{0}=3.4$.

The resolution of the shower position reconstruction in the calorimeter is estimated using the tracker planes. Two detector planes are installed at distances of $86 \mathrm{~mm}$ and $63 \mathrm{~mm}$ in front of the first tungsten plate. Because of the relatively large pad size, about $95 \%$ of the reconstructed clusters in the tracking planes consist of one pad, hence charge sharing between pads cannot be used for the position reconstruction. The impact position of beam particles is set to the middle of the pad. Since the beam particle density is found to be almost constant, a uniform distribution of beam particles within the pitch of the sensor is given. Assuming that the uncertainty of the shower position reconstruction in the calorimeter has a Gaussian distribution, the distributions of the residuals between the particle position in the tracking plane and in the calorimeter is described by the convolution

$f(x)=\frac{B}{p \sigma \sqrt{2 \pi}} \int_{x_{0}-\frac{p}{2}}^{x_{0}+\frac{p}{2}} e^{-\frac{(x-z)^{2}}{2 \sigma^{2}}} \mathrm{~d} z$,

where $\sigma$ is the position resolution in the calorimeter, $p$ the pitch of the tracking plane, $x_{0}$ accounts for relative displace- ment and $B$ provides the normalisation for a given number of events. Figure 17 shows the distribution of the residuals of the reconstructed radial position of the shower in the calorimeter and in the two planes of the tracker. To test the performance of the method the pitch of the sensor can be also considered as a fit parameter. In this case the values found from the fit are $1.86 \mathrm{~mm}$ and $1.71 \mathrm{~mm}$ for the first and second tracking planes, respectively. These numbers are within $5 \%$ equal to the sensor pitch of $1.8 \mathrm{~mm}$. The resolution $\sigma$ of the shower position reconstruction, found from the fit when $p$ is fixed to the value of the sensor pitch, is $(440 \pm 20) \mu \mathrm{m}$, and the absolute values of relative displacements $x_{0}$ are less than $5 \mu \mathrm{m}$.

The small distortion seen at the top part of the distribution for the second tracker plane in Fig. 17 is explained by the small asymmetry of the beam profile and circular geometry of the sensor which, in combination, result in a decline from the uniform distribution of the position uncertainty in the tracking planes.

\subsection{One dimensional transverse shower profile}

The one dimensional profile of the deposited energy in the sensor layers for each event is obtained as the following sum:

$E_{n l}^{d e t}=\sum_{k} \epsilon_{n k l}$

where $\epsilon_{n k l}$ is the deposited energy measured in the sensor pad with radial number $n$, sector $k$ and layer $l$. The sector index $k$ runs over two central sectors of the sensor considered and the layer index $l$ corresponds to the 5 detector planes of the calorimeter. About $5 \%$ of randomly distributed channels in the calorimeter have a larger noise level corresponding to signal sizes of up to 40 MIPs. The influence of these channels, hereafter referred to as bad channels, on the shower development study is eliminated by calculating $\left\langle E_{n l}^{\text {det }}\right\rangle$ for all indexes $n$ and $l$ only from properly working channels.

Since the particle position changes from event to event due to the transverse beam size within about 10 pads, for the estimation of the average value of $\left\langle E_{n l}^{\text {det }}\right\rangle$, the index $n$ in each event is set to $n=0$ for the pad which contains the centre of the shower. An example of the distributions of $E_{n l}^{\text {det }}$ for the shower core $(n=0)$ and pads with $n=-2$, and $n=-5$ for the layer after seven tungsten plates are shown in Fig. 18. Data is well described by the simulation.

\subsection{Longitudinal shower profile}

The average energy $\left\langle E_{l}^{\text {layer }}\right\rangle$ deposited in calorimeter layer $l$ is calculated as the following sum: 


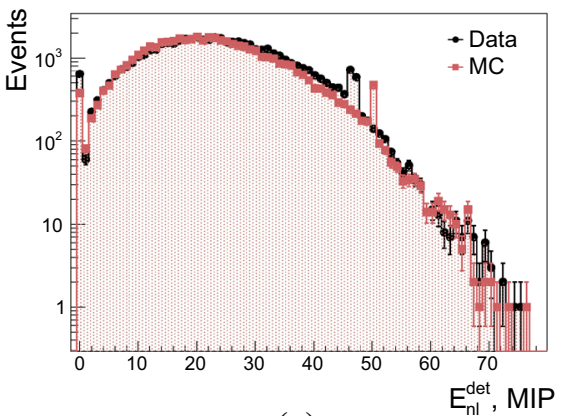

(a)

Fig. 18 Distributions of deposited energy $E_{n l}^{\text {det }}$ as defined in Eq. (7) summed over two sensor sectors L1 and R1 in a layer after seven tungsten plates for radial pads which correspond to shower core (a), two

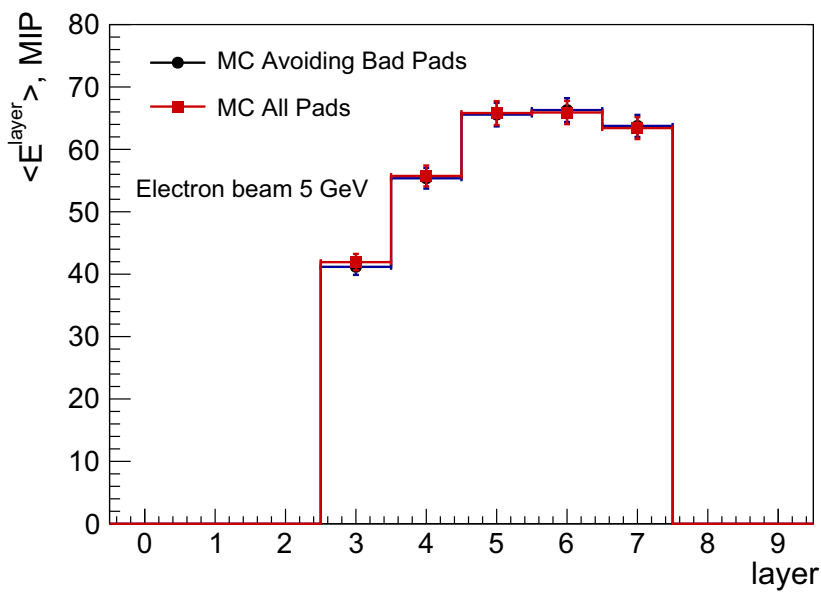

Fig. 19 Longitudinal shower, comparison between simulations with and without bad channels. The distributions are obtained with a $5 \mathrm{GeV}$ electron beam

$\left\langle E_{l}^{\text {layer }}\right\rangle=\sum_{n}\left\langle E_{n l}^{\text {det }}\right\rangle$

where $n$ runs over the radial pads of the two central sectors of the sensor. About 5\% of randomly distributed channels in the calorimeter have a larger noise level corresponding to signal sizes of up to 40 MIPs.

A Monte Carlo simulation has been done to estimate the impact of bad channels on the longitudinal shower profile. The result is shown in Fig. 19. The red distribution corresponds to a calorimeter without bad channels and the black one is obtained after dropping bad channels, introduced in the simulation in the same locations as observed in data. Both distributions agree very well within statistical uncertainties.

The development of the longitudinal shower profile is then measured using only events with properly working channels. In Fig. 20 the deposited energy as a function of the layer $l$ is shown for data and Monte Carlo simulation. The maximum of the shower is reached in data at layer 7 . Both dis-

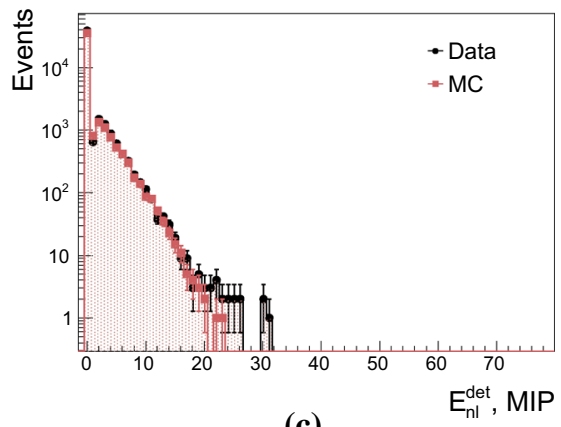

(c)

pads away from the core (b) and five pads away from the core (c). The distributions are obtained with a $5 \mathrm{GeV}$ electron beam

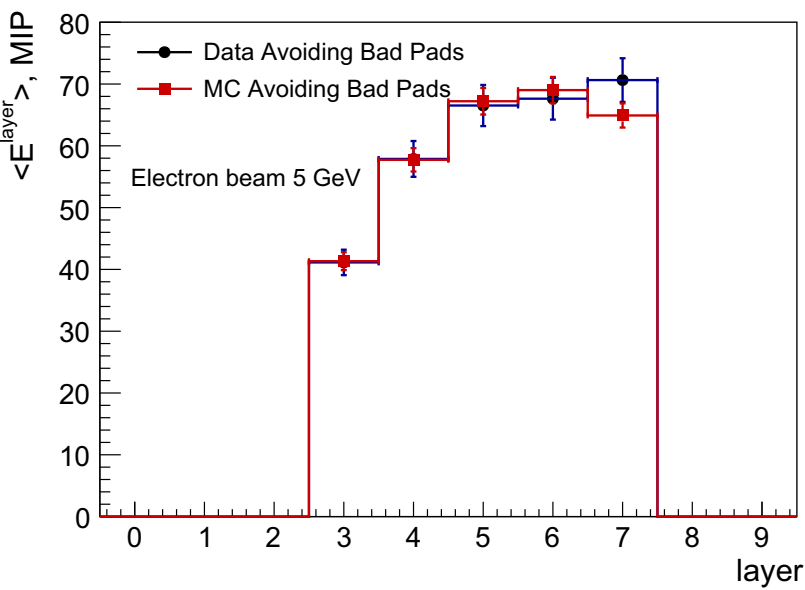

Fig. 20 Longitudinal shower profile, comparison between data and simulation. The distributions are obtained with a $5 \mathrm{GeV}$ electron beam

tributions are, within statistical uncertainties, in reasonable agreement.

\subsection{The Molière radius}

The sensor in Fig. 2 has a relatively fine segmentation in the radial direction, with a pitch of $1.8 \mathrm{~mm}$, but the size of the sectors is between 2 and $2.5 \mathrm{~cm}$ in the irradiated area. Such a geometry does not allow to uniformly sample the electromagnetic shower in the transverse plane and requires the development of a dedicated method to measure the effective Molière radius. Such a method was developed and presented in detail in Ref. [6]. Since here the same sensors are used, this method will be applied. It is briefly described in the following with small modifications which are mainly motivated by the difference in the design of the detector plane.

Denoting $F_{E}(r)$ the density function of the average deposited energy in the transverse plane with respect to the shower axis, the energy in the area covered by a single detector pad can be expressed as the integral 
$E_{n}=\int_{S_{n}} F_{E}(r) \mathrm{d} S$

where $S_{n}$ is the area which corresponds to the sensor pad $n$. The function $F_{E}(r)$ is cylindrically symmetric with respect to the shower axis, and is expressed in cylindrical coordinates with the origin at the center of the shower. Hence it depends only on the radius $r$. Since, on average, $90 \%$ of the deposited energy lies inside a cylinder with a radius of one Molière radius $R_{\mathcal{M}}$, the following equation can be used for the Molière radius calculation:

$0.9=\frac{\int_{0}^{2 \pi} d \varphi \int_{0}^{R_{\mathcal{M}}} F_{E}(r) r \mathrm{~d} r}{\int_{0}^{2 \pi} d \varphi \int_{0}^{\infty} F_{E}(r) r \mathrm{~d} r}$.

The values of $E_{n}$ can be calculated using a parameterised trial functions $F_{E}(r)$. Fitting this trial function to the average deposited energy measured in the corresponding pads, one can define their parameters and use them in Eq. (10) to obtain the Molière radius.

In the previous paper [6], the circular shape of the pads was approximated for simplicity by a straight strip. The effect of this approximation was studied in a simulation [17] and it was shown that the difference between values of $E_{n}$ calculated for pads of circular shape and for strip-like pads depends on the pad position with respect to the shower centre amounts to at most $2 \%$. This difference was included in the systematic uncertainty. This effect was also diminished in the data analysis because the detector planes had limited number of pads connected to the readout and some values of $E_{n}$ could not be measured directly, but were recovered assuming the symmetry with respect to the shower core.

In the present study, the numerical integration in Eq. (9) is done using the correct geometry of the sensor pad. To this end it is convenient to use cylinder coordinates which are linked to the sensor geometry. Changing the coordinates to $\mathbf{r}=\mathbf{r}^{\prime}-\mathbf{R}_{\mathbf{0}}^{\prime}$, where $\mathbf{R}_{0}^{\prime}$ is the position of the shower axis in the sensor reference frame, the pad energy can be obtained by the integration:

$E_{n}=\int_{\varphi_{\min }^{\prime}}^{\varphi_{\max }^{\prime}} \int_{r_{n}^{\prime}}^{r_{n+1}^{\prime}} F_{E}\left(\left|\mathbf{r}^{\prime}-\mathbf{R}_{\mathbf{0}}^{\prime}\right|\right) r^{\prime} \mathrm{d} r^{\prime} \mathrm{d} \varphi^{\prime}$,

where $\varphi_{\min }^{\prime}$ and $\varphi_{\max }^{\prime}$ correspond to the sectors of the sensor and $r_{n}^{\prime}$ to the radius of the sensor pad $n$. The integration over $\varphi^{\prime}$ comprises the sectors L1 and R1 (see Fig. 2) which corresponds to about $40 \mathrm{~mm}$. Since the transverse size of the beam is $\sigma_{x, y} \approx 4.2 \mathrm{~mm}$ and the expected effective Molière radius is around $10 \mathrm{~mm}$, the two sectors safely cover one effective Molière radius of the shower.
The trial function used to describe the average transverse energy profile of the shower is a Gaussian for the core, dominated by the high energy component of the shower, and a form inspired by the Grindhammer-Peters parameterisation $[18,19]$ to account for the tails originating from the low energy photon halo,

$F_{E}(r)=A_{C} e^{-\left(\frac{r}{R_{C}}\right)^{2}}+A_{T} \frac{2 r^{\alpha} R_{T}^{2}}{\left(r^{2}+R_{T}^{2}\right)^{2}}$,

where $A_{C}, R_{C}, A_{T}, R_{T}$ and $\alpha$ are parameters to be determined by fitting the function to the measured distribution.

As can be seen from Eq. (11), the energy $E_{n}$ deposited in the pad number $n$ depends on the shower position $\mathbf{R}_{\mathbf{0}}^{\prime}$ and pad position $\mathbf{r}_{\mathbf{n}}^{\prime}$. Since the beam transverse size is significantly smaller than the radius $R_{0}^{\prime}$, the calculation of $E_{n}$ is done for a value of $R_{0}^{\prime}$ which corresponds to the position of the maximum in the beam profile. This maximum is observed in a pad with $n=45$ and $r_{n}^{\prime}=161 \mathrm{~mm}$.

\subsection{The effective Molière radius determination at $5 \mathrm{GeV}$}

The average profile of the electromagnetic transverse shower is determined by summing over all detector layers,

$\left\langle E_{n}^{\text {det }}\right\rangle=\sum_{l}\left\langle E_{n l}^{\text {det }}\right\rangle$.

The measured averaged transverse energy values, $\left\langle E_{n}^{\text {det }}\right\rangle$, were fitted to the function in Eq. (12). Results for data and Monte Carlo simulation for electrons of $5 \mathrm{GeV}$ energy, are shown in Fig. 21, where one sees the dependence of $\left\langle E_{n}^{\text {det }}\right\rangle$ on the distance from the shower core, $d_{\text {core }}$. The simulation agrees well with the data.

The fitted function reproduces the experimental and the simulated transverse shower profile with an accuracy better than 5\%. Fig. 22 shows the right part of Eq. (10) as a function of the radial integration limit $R$ for data and simulation with the horizontal line demonstrating a graphical solution for the effective Molière radius. The result is $(8.1 \pm 0.1$ (stat) \pm 0.3 (syst)) $\mathrm{mm}$, a value well reproduced by the MC simulation $(8.4 \pm 0.1) \mathrm{mm}$. The result obtained here is much smaller than the one determined in the calorimeter prototype used during the 2014 test beam with larger gaps between the tungsten plates, which yielded $(24.0 \pm 1.6) \mathrm{mm}[6]$.

\subsection{Energy dependence of the effective Molière radius}

The main analysis was performed for data taken at $5 \mathrm{GeV}$ beam energy. In addition, data were taken for energies between 1 and $5 \mathrm{GeV}$. For the study of the energy dependence, about 50,000 events were used for each energy, and the measurement of the effective Molière radius was carried out as for the $5 \mathrm{GeV}$ sample. An example of the average 


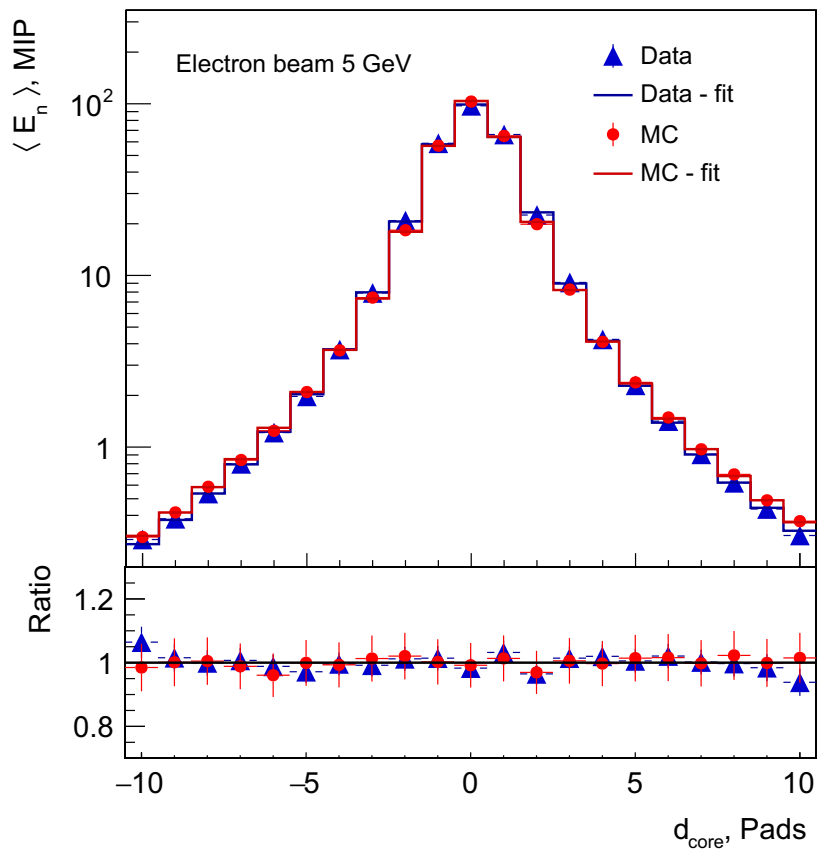

Fig. 21 The average transverse shower profile, $\left\langle E_{n}^{\text {det }}\right\rangle$, as a function of the distance from the core, $d_{\text {core }}$, in units of the pad dimension $(1.8 \mathrm{~mm})$, for data (blue triangles) and MC simulation (red circles). The histograms are the results of fits to data and Monte Carlo simulation to the function in Eq. (12). The distributions are obtained with a 5 $\mathrm{GeV}$ electron beam. The lower part of the figure shows the ratio of the distributions to the fitted function, for the data (blue) and simulation (red)

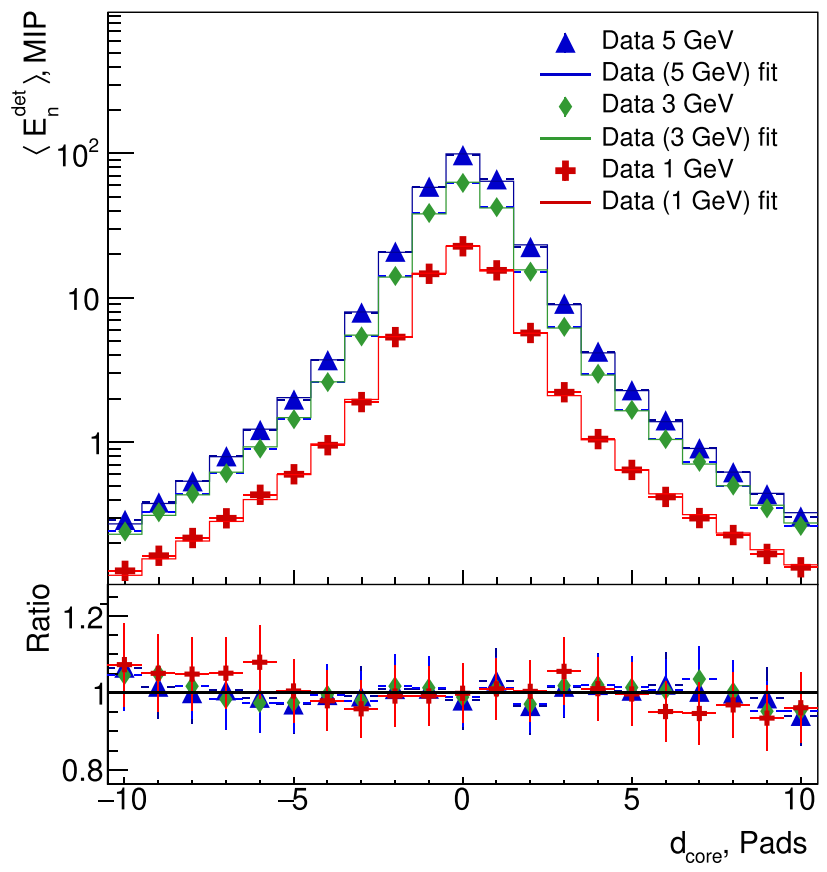

Fig. 23 Average transverse shower profiles for 1,3 and $5 \mathrm{GeV}$ electrons in data and the ratios between data and the fitted functions

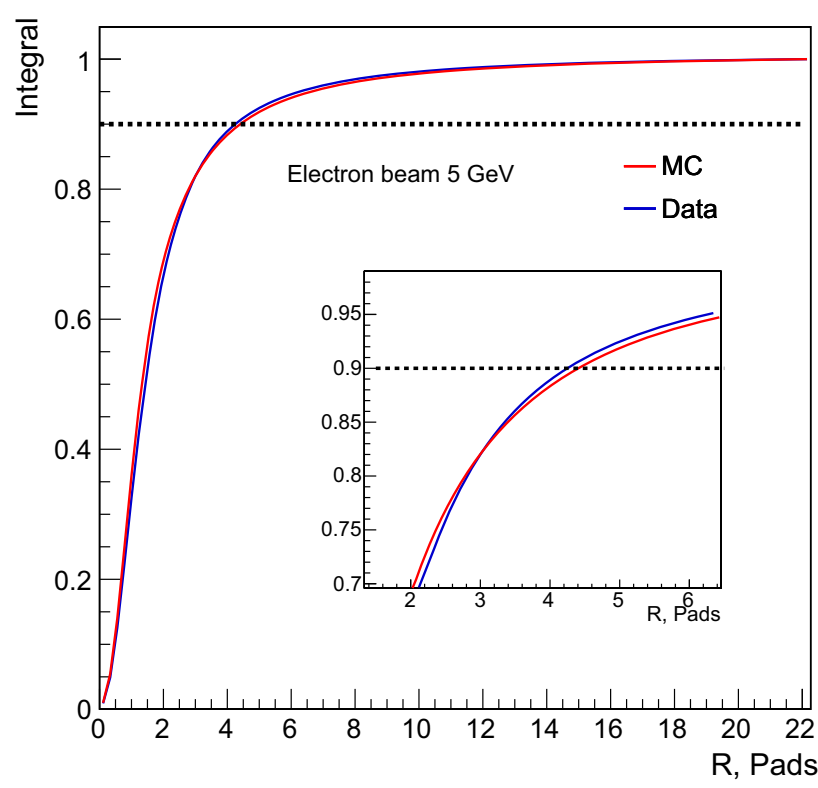

Fig. 22 The ratio of the integrals in Eq. (10) using $F_{E}(r)$ obtained from the fit, as a function of the radius $R$ in units of the pad dimension $(1.8 \mathrm{~mm})$, for data (blue) and $\mathrm{MC}$ (red), for a $5 \mathrm{GeV}$ electron beam. The insert shows an expanded view of the region $2<R<6$ pads

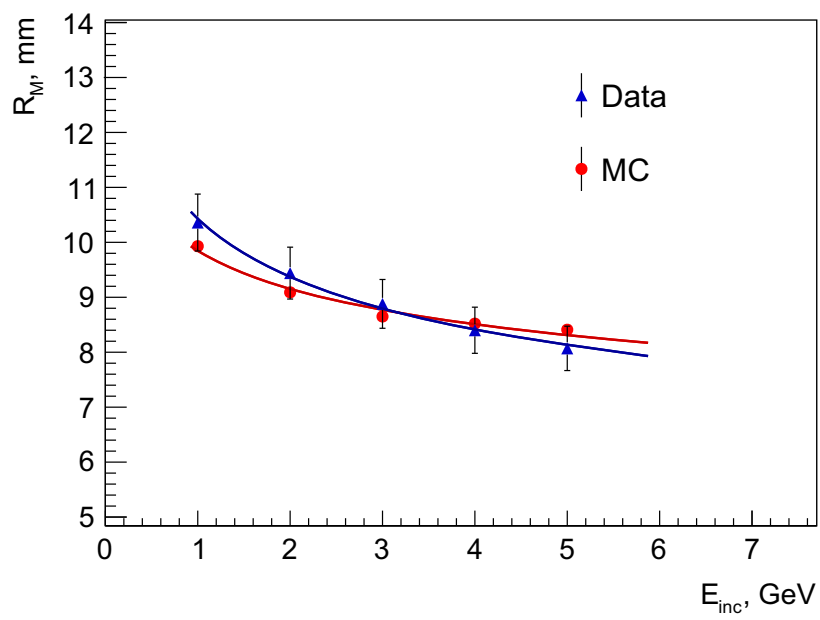

Fig. 24 The effective Molière radius as a function of the electron energy for data (blue) and simulation (red) 


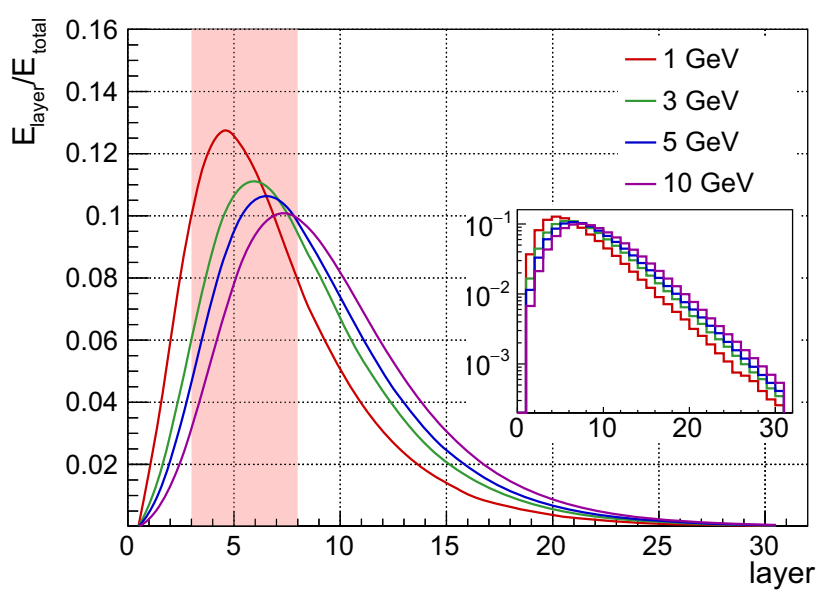

Fig. 25 Normalised longitudinal shower profile for different electron energies obtained in simulation. Highlighted region corresponds to sensitive layers installed in the calorimeter. The inset shows the same profiles in a logarithmic scale

transverse shower profiles at 1,3 and $5 \mathrm{GeV}$ beam energy is shown in Fig. 23. The average deposited energies are lower at lower beam energies, and the distributions are wider, resulting in a larger value of the effective Molière radius. The data are again well described by the results of simulations.

The effective Molière radius as a function of the incoming electron energy, $E_{i n c}$, in the range of $1-5 \mathrm{GeV}$ is shown in Fig. 24. It decreases with the electron energy as $E_{\text {inc }}^{(-0.15 \pm 0.04)}$. The fit to the simulation yields an exponent $(-0.11 \pm 0.01)$, in agreement with the data.

In order to investigate the observed energy dependence of the effective Molière radius, a simulation of an "infinite" calorimeter was performed. In practice the simulated calorimeter consisted of 30 planes with transverse size of $40 \times 40 \mathrm{~cm}^{2}$. Absorbers, detector layers and gaps had the same composition and thickness as the ones of the tested prototype.

Figure 25 shows the normalised average longitudinal profile of the energy deposited in the detector layers for incident electrons of different energy. The depth of the calorimeter is sufficient to contain most of the shower even for $10 \mathrm{GeV}$ electrons in which case the fraction of the energy deposited in the last sensor layer is below $0.3 \%$, as can be seen in the insert in Fig. 25. The detector layers from 3 to 7, as installed in the prototype (shaded area) probe different regions of the longitudinal shower profile for different energies. For $1 \mathrm{GeV}$ electrons, the shower is measured almost symmetrically around its maximum, while for $5 \mathrm{GeV}$ electrons the layers 3-7 cover mostly the left side from the maximum. Hence, the fraction of the energy recorded in these layers depends on the beam energy. In Fig. 26 the cumulative distribution of the fraction of the deposited energy is shown as a function of the number of layers. In layers 3 to 7 , the fractions for $1 \mathrm{GeV}, 3 \mathrm{GeV}$

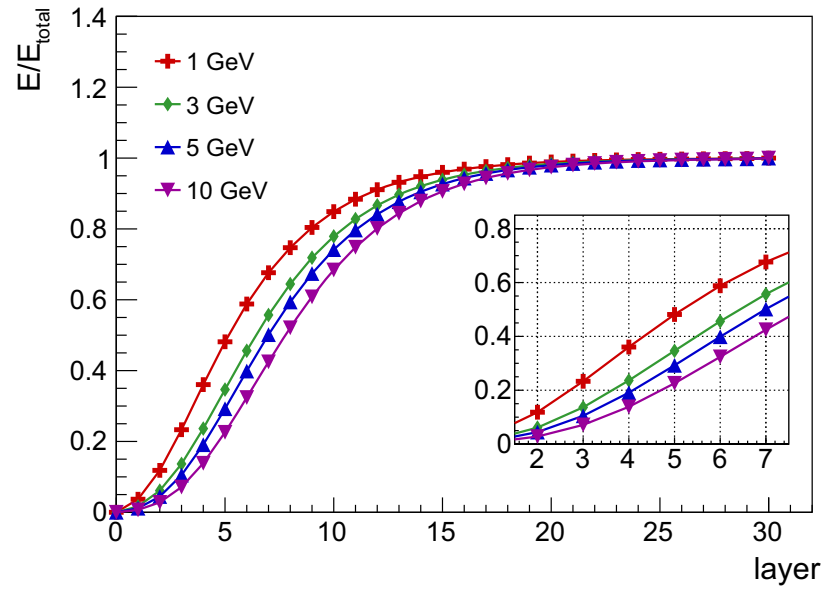

Fig. 26 Cumulative distribution of the fraction of energy deposited in the detector layers as a function of the number of layers for different electron beam energies. The insert shows an expanded view of the region for planes 2 to 7

and $5 \mathrm{GeV}$ electrons are $56 \%, 50 \%$ and $46 \%$, respectively. This difference explains a small deviation from linearity in the observed prototype response as was shown in Fig. 16 with red line and squares. Those measurements corrected to represent equal fractions of beam energies are shown with blue triangles and they are in good agreement with a linear fit.

The measurement of the shower in fixed detector layer positions for different longitudinal shower profiles also influences the observed transverse shower size. As can be seen from Fig. 27, the RMS of the lateral projection of the deposited energy in each detector layer is expected to increase as a function of the sensor layer number, with a steeper slope for lower electron energies. The small increase of the RMS observed in the first and second layers are explained by the back-scattering of shower particles. According to the results in Fig. 27 it is expected that the effective Molière radius decreases with increasing beam energy for the beam test geometry. When the fraction of the sampled shower energy approaches unity for different electron energies, the Molière radii converge to the same value. This can be seen in Fig. 28, where the calculated Molière radius is shown as a function of the number of detector layers included in the calculation. Thus, the observed dependence of the effective Molière radius in the prototype on the incident electron energy, as presented in Fig. 24, is due to the limited number of detector layers installed near the shower maximum. The slightly higher values of the effective Molière radius observed in the simulated calorimeter originate from the fact that in the simulation the transverse size of the calorimeter was much larger than that of the prototype. The difference is well reproduced by the simulation. 


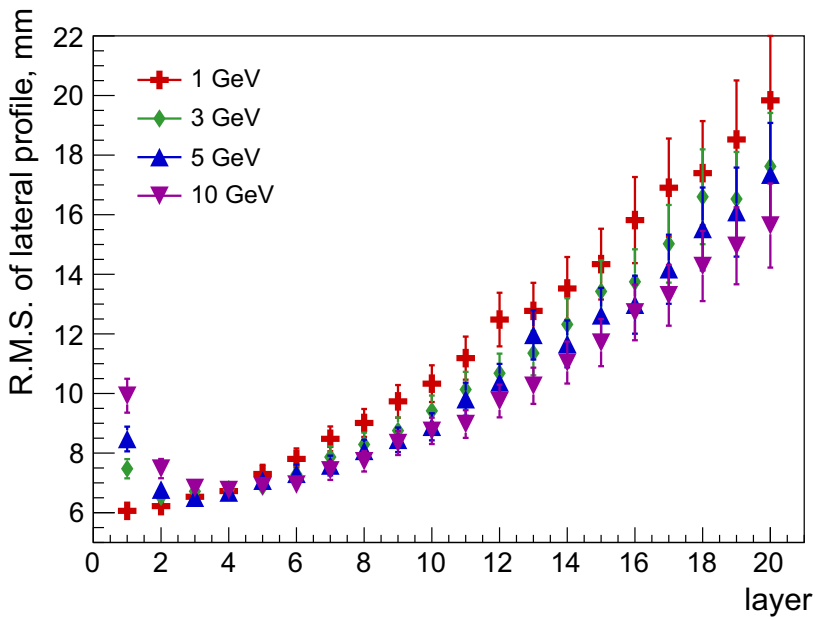

Fig. 27 RMS of the lateral deposited energy distributions in detector layers obtained in simulation, for different electron beam energies

\subsection{Uncertainties}

The study of the systematic uncertainty of the measured average energy deposition in the transverse direction $\left\langle E_{n l}^{\text {det }}\right\rangle$ includes the following contributions:

- uncertainty of the measured efficiency of the signal identification;

- uncertainty of the particle impact position measurement and misalignment of detector planes;

- uncertainty due to bad channels;

- noise uncertainty;

- calibration uncertainty.

The uncertainty due to the efficiency of the signal reconstruction is evaluated by changing the efficiency according to high and low edges of the shaded area in Fig. 14. The result for the effective Molière radius changes by $\pm 0.16 \mathrm{~mm}$.

The misalignment of the detector planes is estimated using occupancy plots for each layer. It is accounted for in the geometry of the simulation. The effect of misalignment on the effective Molière radius comes from the sum in Eq. (13) where the radial pad index $n$ denotes pads in different layers which are assumed to be aligned in the longitudinal direction. Due to misalignment, the average lateral deposited energy $\left\langle E_{n}^{\text {det }}\right\rangle$ for a given distance from the shower core, determined by the index $n$, gets contribution from pads which are at different distances from the shower core. A similar effect arises from the uncertainty of the particle impact position. This uncertainty is estimated by calculating the effective Molière radius from simulations with perfectly aligned sensors and sensors displaced within the estimated misalign-

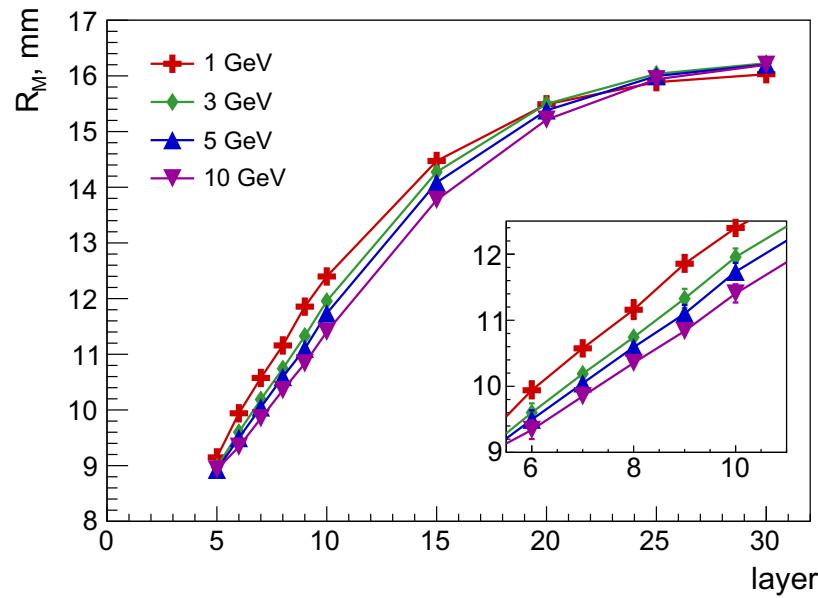

Fig. 28 Molière radius, obtained in simulation, for different electron beam energies as a function of number of detector layers in the calorimeter. The insert shows an expanded view of the plane region 6-10

ment. The change of the effective Molière radius is found to be $0.08 \mathrm{~mm}$.

The influence of the bad channels, which are included into simulation, leads to a change of the effective Molière radius by $0.14 \mathrm{~mm}$ compared to the simulation where all channels work properly.

The effect due to the usage of one single radius $R_{0}^{\prime}$ in Eq. (11) for the calculation of $E_{n}$ is estimated by selecting a narrow range of the particles impact position around the sensor pad with the radial index $n=45$. The relative change of the effective Molière radius is within $0.13 \mathrm{~mm}$.

The contribution of the measured noise uncertainty was studied in the simulation and found to be significantly below $1 \%$.

A relative calibration uncertainty of 5\% for each APV25 front-end board is assigned to each value $\left\langle E_{n l}^{\text {det }}\right\rangle$ in Eq. (13) and summed in quadrature to determine the uncertainty of $\left\langle E_{n}^{d e t}\right\rangle$. The calibration uncertainty is combined with the statistical one and used to produce 1000 transverse shower profiles where each $\left\langle E_{n}^{\text {det }}\right\rangle$ is randomly generated using a Gaussian distribution function with a mean value corresponding to the measured $\left\langle E_{n}^{d e t}\right\rangle$ and a $\sigma$ determined by the uncertainty. For each shower, the effective Molière radius is calculated and the RMS of their distribution is considered as a contribution to the statistical uncertainty of the effective Molière radius measurement.

The contributions to the systematic uncertainty are considered to be independent. The total systematic uncertainty on the Molière radius measurement is obtained by adding all the contributions in quadrature. 


\section{Summary and conclusions}

New sub-millimeter thickness detector layers for the luminosity calorimeter LumiCal have been designed and produced. Silicon sensors are read out using Kapton fan-outs with copper traces connected via wire bonding or TAB to the sensor pads. The eight assembled detector layers were installed in the $1 \mathrm{~mm}$ gap between the tungsten absorber plates and successfully operated during the 2016 beamtest campaign. Measurements of the shower position and the longitudinal and transverse shower shape are presented and compared to Monte Carlo simulations. The effective Molière radius of this compact calorimeter prototype was determined at $5 \mathrm{GeV}$ to be $(8.1 \pm 0.1$ (stat) \pm 0.3 (syst) $) \mathrm{mm}$, a value well reproduced by the MC simulation $(8.4 \pm$ $0.1) \mathrm{mm}$. Its energy dependence in the range $1-5 \mathrm{GeV}$ was also studied. The observed slight decrease proportional to $E_{\text {inc }}^{(-0.15 \pm 0.04)}$, can be explained by the limited number of detector planes used to probe the electromagnetic shower.

These results demonstrate the feasibility of constructing a compact calorimeter consistent with the conceptual design, which is optimised for a high precision luminosity measurement in future $\mathrm{e}^{+} \mathrm{e}^{-}$collider experiments.

Acknowledgements This study was partly supported by the Israel Science Foundation (ISF), Israel German Foundation (GIF), the ICORE program of the Israel Planning and Budgeting Committee, Israel Academy of Sciences and Humanities, by the National Commission for Scientific and Technological Research (CONICYT - Chile) under Grant FONDECYT 1170345 and PIA/Basal FB0821, by the Polish Ministry of Science and Higher Education under Contract rs 3585/H2020/2016/2 and 3501/H2020/2016/2, the Rumanian UEFISCDI agency under contracts PN-II-PT-PCCA-2013-4-0967 and PN-II-ID-PCE-2011-3-0978, by the Ministry of Education, Science and Technological Development of the Republic of Serbia within the project O1171012, by the United States Department of Energy, grant DE-SC0010107, and by the European Union Horizon 2020 Research and Innovation programme under Grant Agreement No. 654168 (AIDA-2020). The measurements leading to these results have been performed at the Test Beam Facility at DESY Hamburg (Germany), a member of the Helmholtz Association (HGF).

Data Availability Statement This manuscript has no associated data or the data will not be deposited. [Authors' comment: Test data are usually not deposited. Please contact the corresponding author for details.]

Open Access This article is distributed under the terms of the Creative Commons Attribution 4.0 International License (http://creativecomm ons.org/licenses/by/4.0/), which permits unrestricted use, distribution, and reproduction in any medium, provided you give appropriate credit to the original author(s) and the source, provide a link to the Creative Commons license, and indicate if changes were made.

Funded by SCOAP ${ }^{3}$.

\section{References}

1. H. Abramowicz et al., Forward instrumentation for ILC detectors JINST 5, P12002 (2010)

2. T. Behnke et al., The International Linear Collider. Technical Design Report, Volume 4: Detectors (2013). arXiv:1306.6329 [physics.ins-det]

3. H. Baer et al., The International Linear Collider. Technical Design Report, Volume 2: Physics. (2013). arXiv:1306.6352 [hep-ph]

4. The CLIC, CLICdp collaborations, Updated baseline for a staged Compact Linear Collider. CERN Yellow Report CERN-2016-004. arXiv: 1608.07537 [physics.acc-ph]

5. D. Bardin et al., One-loop electroweak radiative corrections to polarized Bhabha scattering. arXiv:1801.00125 [hep-ph]

6. H. Abramowicz et al., Measurement of shower development and its Molière radius with a four-plane LumiCal test set-up. Eur. Phys. J. C 78, 135 (2018). arXiv:1705.03885. https://doi.org/10.1140/epjc/ s10052-018-5611-9

7. F.-X. Nuiry, Collected documents on the FCAL-AIDA precision mechanical infrastructure and tungsten plates. https://edms.cern. $\mathrm{ch} /$ document/1475879/

8. M. Raymond et al., The APV25 $0.25 \mu \mathrm{m}$ CMOS readout chip for the CMS tracker. IEEE Nucl. Sci. Symp. Conf. Rec. 2, 9/113 (2000)

9. M.J. French et al., Design and results from the APV25, a deep sub-micron CMOS front-end chip for the CMS tracker. Nucl. Instr. Methods A 466, 359 (2001)

10. S. Martoiu, H. Muller, J. Toledo, Front-end electronics for the Scalable Readout System of RD51. Proc. IEEE Nucl. Sci. Symp. Conf. Rec., Valencia pp. 2036-2038 (2011). https://doi.org/10. 1109/NSSMIC.2011.6154414

11. H. Abramowicz et al., Performance of fully instrumented detector planes of the forward calorimeter of a Linear Collider detector. JINST 10, P05009 (2015)

12. M. Bregant et al., Assembly and validation of the ALICE silicon microstrip detector. Nucl. Instr. Methods A 570, 312 (2007)

13. S. Martoiu, H. Muller, A. Tarazona, J. Toledo. Development of the scalable readout system for micro-pattern gas detectors and other applications. Topical Workshop on Electronics for Particle Physics 2012, 17-21 September 2012, OXFORD, U.K. JINST 8, C03015 (2013)

14. G.A. Akopdjanov et al., Determination of photon coordinates in a hodoscope Cherenkov spectrometer. Nucl. Instr. Methods 140, 441 (1977)

15. L. Bugge, On the determination of shower central positions from lateral samplings. Nucl. Instr. Methods A 242, 228 (1986)

16. T.C. Awes, F.E. Obenshain, F. Plasil, S. Saini, S.P. Sorensen, G.R. Young, A simple method of Shower Localization and Identification in Laterally Segmented Calorimeters. Nucl. Instr. Methods A 311, 130 (1992)

17. O. Borysov for the FCAL collaboration, Beam Tests of a Multilayer LumiCal Prototype. Proceedings of the International Workshop on Future Linear Colliders - LCWS2016, Morioka, Japan, December 5-9, 2016. arXiv:1703.09955 [physics.ins-det]

18. G. Grindhammer et al., Proceedings of the Workshop on Calorimetry for the Supercollider, Tuscaloosa, AL, March 13-17, 1989, edited by R. Donaldson and M.G.D. Gilchriese (World Scientific, Teaneck, NJ, p. 151) (1989)

19. G. Grindhammer, M. Rudowicz, S. Peters. The parameterized simulation of electromagnetic showers in homogeneous and sampling calorimeters. arXiv:hep-ex/0001020 